\title{
Magnetic resonance neurography: is it so complicated that it needs a touch of genius?
}

\author{
Ankita Aggarwal ${ }^{1} \cdot$ Avneesh Chhabra ${ }^{2,3,4,5}$
}

Received: 2 November 2021 / Revised: 2 November 2021 / Accepted: 10 November 2021 / Published online: 1 February 2022

(c) The Author(s), under exclusive licence to European Society of Radiology 2022

\section{Key Points}

- Neuropathy imaging is not that complicated or illusive that it needs a touch of genius.

- By gaining MR imaging expertise of peripheral nerve lesions and using knowledge of common clinical patterns and diseases, general radiology practitioners can prudently participate in the multidisciplinary care for appropriate and timely management of peripheral neuropathy patients.

Imaging of peripheral nerves seems challenging owing to their small calibre, proximity to the vessels, frequent oblique course, and complex anatomy. Magnetic resonance neurography (MRN) is an advancement of MR technique devised specifically to highlight the peripheral nerve anatomy, architecture, and its pathology along its longitudinal axis with superior resolution akin to MR angiography for vessels [1]. Every year almost $5 \%$ of the population is affected by peripheral neuropathy with a relatively higher frequency in the elderly. The technique of MRN has vastly improved over the years, largely due to wider availability of higher strength and quality magnet scanners as well as innovations in new sequence developments [2] with time-efficient isotropic 3D (dimensional) imaging producing spin-echo type contrast resolution. MRN has been found to be clinically valuable leading to impact in decision making and treatments for neuropathies due to traumatic injuries, entrapment, neoplasm characterization, and post-operative follow-up [3]. In tertiary care centres like ours, diffusion-weighted imaging is routinely used in conjunction with $3 \mathrm{D}$ fat-suppressed MRN

Avneesh Chhabra

avneesh.chhabra@utsouthwestern.edu

1 Radiology, VMMC \& Safdarjung Hospital, Delhi, India

2 Radiology \& Orthopaedic Surgery, UTSW, TX 75390-9178 Dallas, USA

3 Musculoskeletal Radiology UT Southwestern Medical Centre, 5323 Harry Hines Blvd, TX, Dallas, USA

4 Johns Hopkins University, Baltimore, MD, USA

5 Walton Centre for Neuroscience, Liverpool, UK that enables the generation of vessel signal suppressed MR neurograms for excellent depiction of nerve pathology.

The term MRN was coined in the early 1990s, and the technique then described primarily included a combination of T1 W and 2D STIR (short tau inversion recovery) images $[4,5]$. However, the beginning was not easy with many hurdles. Lower magnetic strength scanners at that time generated poor image resolution, low contrast to noise ratio, inadequate fat- and vascular-signal suppression, and exhibited longer scanning times. The advent of 3-Tesla scanner, improved phased-array surface coils, and parallel imaging have revolutionized the MRN technique [6]. 3-D isotropic technique using 3D-SITR and 3D-PSIF (reversed imaging in steady state free precession), and diffusion tensor imaging (DTI) when used in combination with axial $\mathrm{T} 1 \mathrm{~W}$ and axial T2 SPAIR or T2 Dixon imaging provides comprehensive evaluation of the regional neuromuscular structures aiding in both qualitative and quantitative assessments. Qualitative assessment like any other organ system includes evaluation of peripheral nerve contour, calibre, signal, fascicular disruption, and intralesional fat, etc. Quantitative assessment involves evaluation of parameters like fractional anisotropy (FA), apparent diffusion coefficient (ADC), and contrast enhancement [7].

MRN technique has immensely benefited the neuropathy patients, e.g. preoperative determination of the Sunderland injury grade, distinguishing diabetic amyotrophy from radiculopathy, detecting diffuse neuropathy condition like Charcot Marie tooth disease or chronic demyelinating polyneuropathy, characterizing nerve tumour as benign or malignant, finding exact site of nerve entrapment or demonstrating the post-tunnel release re-entrapment, and in addition, 


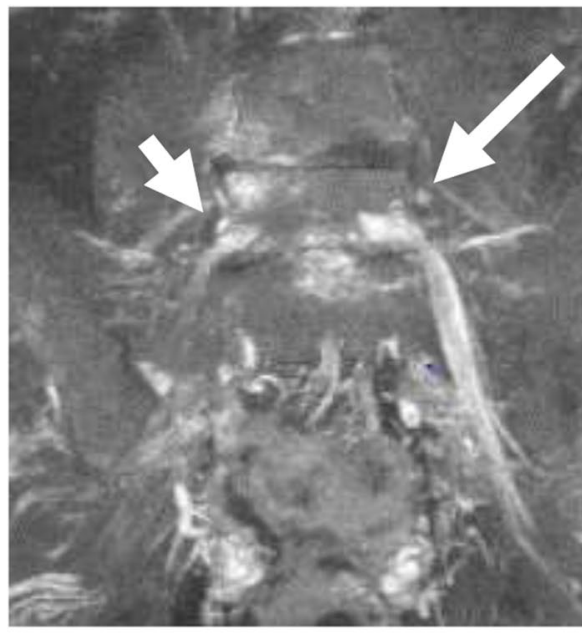

a

Fig. 1 Left lower back and buttock pain after recent sudden exertion. MR imaging of lumbar spine revealed L5-S1 osteophytes and disc bulge (not shown). (a) Grey scale and (b) colour heat map MR neurography of lumbosacral plexus using 3D STIR sequence with maximum intensity projection revealed focal constriction and torsion

determining the regional muscle denervation changes, etc. [6-9].

The major question in vogue is whether such concepts of MRN can be applied to the routine practice by general radiologists or is it still a forte of the nerve imaging sub-specialists? The authors strongly believe that in lieu of availability of abundant literature, conventional radiologists can adapt the interpretation principles in their practices. 3D imaging is also becoming routine on $1.5 \mathrm{~T}$ and $3 \mathrm{~T}$ scanners. Main hurdle seems to be the lack of interest and efforts needed in gaining clinical and imaging knowledge by radiologists in the domain of peripheral neuropathy. While ultrasound is popular, especially in European countries for peripheral nerve imaging, it requires operator expertise, and deeper nerves are challenging to interrogate. Using conventional highresolution multiplanar MR imaging, finding the precise site of injury, grading nerve injury to aid pre-operative assessment for the nerve surgeon, characterizing nerve tumours, detecting lesions, such as multi-loculated elongated intraneural ganglion, classic honeycomb pattern of a perineurioma, or hourglass constriction of severe entrapment is not that difficult. MRN though has the added advantage of displaying these pathologies in the long-axis adding to the diagnostic confidence level of the reader, e.g. for finding nerve torsion (Fig. 1) [9]. In fact, pudendal, superficial peroneal and smaller sensory nerves like lateral or medial antebrachial cutaneous and radial sensory nerves are best seen on axial 2D imaging, though MRN is ideally suited for fine craniofacial nerves of left L5 nerve with proximal and distal enlargement and asymmetric hyperintensity and yellow colouration (large arrows) compared to normal right L5 nerve root (small arrows). The patient improved gradually following CT-guided left L5 perineural injection of anaesthetic and steroid

like for facial, lingual, or occipital pain using 3D PSIF or 3D DESS MR imaging.

To describe findings seen in MRN, structured reporting is ideally used, as shown by Chhabra et al in dedicated books on MRN and musculoskeletal MRI structured evaluation. General practitioners can adopt similar practice for detailed description of MR imaging findings in their reports. The reader should treat peripheral neuromuscular structures as another organ system in question in the field of view, i.e. assess neuromuscular signal, size, contour, and intra-lesional haemorrhage and fat. One should also determine and report the possible aetiology of neuropathy, e.g. entrapment or injury rather than just calling the nerve hyperintense and possible neuropathy. In future, standardized lexicon development, such as neuropathy score reporting and data system, may facilitate more standardized reporting like BIRADS. Further refinement and improved post-processing of techniques of diffusion-weighted imaging will help its widespread application, since these images are very sensitive for qualitative assessment of neuropathy and, in addition, generate quantification parameters as described above.

Thus, neuropathy imaging is not that complicated or illusive that it needs a touch of genius. By gaining MR imaging expertise of peripheral nerve lesions and using knowledge of common clinical patterns and diseases, general radiology practitioners can prudently participate in the multidisciplinary care for appropriate and timely management of peripheral neuropathy patients. Such an approach can tremendously benefit their clinical care with potential positive outcomes and prognosis. 
Funding The authors state that this work has not received any funding.

\section{Declarations}

Guarantor The scientific guarantor of this publication is Dr. Avneesh Chhabra.

Conflict of interest AC: consultant: ICON Medical and TREACE Medical Concepts Inc., Book Royalties: Jaypee, Wolters, speaker: Siemens, Medical advisor: Image biopsy Inc.The remaining authors of this manuscript declare no relationships with any companies whose products or services may be related to the subject matter of the article.

Statistics and biometry Not applicable.

Informed consent Not applicable.

Ethical approval Not applicable.

Methodology • Editorial

\section{References}

1. Bunch PM (2018) Invited Commentary on "MR Neurographic Evaluation of Facial and Neck Pain." Radiographics 38:15131515. https://doi.org/10.1148/rg.2018180144

2. Wang X, Harrison C, Mariappan YK et al (2017) MR neurography of brachial plexus at $3.0 \mathrm{~T}$ with robust fat and blood suppression.
Radiology 283:538-546. https://doi.org/10.1148/radiol.20161 52842

3. Chhabra A, Belzberg AJ, Rosson GD et al (2016) Impact of high resolution 3 tesla MR neurography (MRN) on diagnostic thinking and therapeutic patient management. Eur Radiol 26:1235-1244. https://doi.org/10.1007/s00330-015-3958-y

4. Filler AG, Kliot M, Howe FA et al (1996) Application of magnetic resonance neurography in the evaluation of patients with peripheral nerve pathology. J Neurosurg 85:299-309. https://doi.org/10. 3171/jns.1996.85.2.0299

5. Howe FA, Filler AG, Bell BA, Griffiths JR (1992) Magnetic resonance neurography. Magnet Reson Med 28:328-338. https://doi. org/10.1002/mrm.1910280215

6. Chhabra A, Andreisek G, Soldatos T et al (2011) MR neurography: past, present, and future. AJR Am J Roentgenol 197:583591. https://doi.org/10.2214/AJR.10.6012

7. Hlis R, Poh F, Bryarly M et al (2019) Quantitative assessment of diabetic amyotrophy using magnetic resonance neurography-a case-control analysis. Eur Radiol 29:5910-5919. https://doi.org/ 10.1007/s00330-019-06162-3

8. Schwarz D, Weiler M, Pham M et al (2015) Diagnostic signs of motor neuropathy in MR neurography: nerve lesions and muscle denervation. Eur Radiol 25:1497-1503. https://doi.org/10.1007/ s00330-014-3498-x

9. Chalian M, Chhabra A (2019) Top-10 Tips for getting started with magnetic resonance neurography. Semin Musculoskelet Radiol 23:347-360. https://doi.org/10.1055/s-0039-1677727

Publisher's note Springer Nature remains neutral with regard to jurisdictional claims in published maps and institutional affiliations. 